University of Nebraska - Lincoln

DigitalCommons@University of Nebraska - Lincoln

1989

\title{
An Empirical Bayes Approach to Analyzing Recurring Animal Surveys
}

Douglas H. Johnson

USGS Northern Prairie Wildlife Research Center, Douglas_H_Johnson@usgs.gov

Follow this and additional works at: https://digitalcommons.unl.edu/usgsnpwrc

Part of the Other International and Area Studies Commons

Johnson, Douglas H., "An Empirical Bayes Approach to Analyzing Recurring Animal Surveys" (1989). USGS Northern Prairie Wildlife Research Center. 199.

https://digitalcommons.unl.edu/usgsnpwrc/199

This Article is brought to you for free and open access by the US Geological Survey at DigitalCommons@University of Nebraska - Lincoln. It has been accepted for inclusion in USGS Northern Prairie Wildlife Research Center by an authorized administrator of DigitalCommons@University of Nebraska - Lincoln. 


\title{
AN EMPIRICAL BAYES APPROACH TO ANALYZING RECURRING ANIMAL SURVEYS ${ }^{1}$
}

\author{
DOUGLAS H. JOHNSON \\ United States Fish and Wildlife Service, Northern Prairie Wildlife Research Center, \\ Jamestown, North Dakota 58402 USA
}

\begin{abstract}
Recurring estimates of the size of animal populations are often required by biologists or wildlife managers. Because of cost or other constraints, estimates frequently lack the accuracy desired but cannot readily be improved by additional sampling. This report proposes a statistical method employing empirical Bayes (EB) estimators as alternatives to those customarily used to estimate population size, and evaluates them by a subsampling experiment on waterfowl surveys. EB estimates, especially a simple limitedtranslation version, were more accurate and provided shorter confidence intervals with greater coverage probabilities than customary estimates.
\end{abstract}

Key words: animal surveys; empirical Bayes; population estimation; subsampling; waterfowl.

\section{INTRODUCTION}

Many animal populations are surveyed at regular intervals to determine whether they are increasing, decreasing, or remaining relatively constant in size. Such surveys are particularly useful for monitoring threatened species or for surveying game species. Although considerable attention has been given to the design of population surveys (e.g., Eberhardt 1978, Ralph and Scott 1981, Seber 1982, Cooperrider et al. 1986), the analysis of these count data often proceeds with little or no recognition of previous surveys. Yet, earlier surveys can provide valuable data, especially for populations that do not change drastically from one survey occasion to the next. Statistical techniques exist that exploit such auxiliary information as results of earlier surveys to improve population size estimates to a level equivalent to a doubling of the sample size, which is often limited by financial or logistic constraints. One such technique is empirical Bayes (EB) estimation, which has been successfully applied in several fields (e.g., Wilcox [1977] for educational testing, Harris and Shakarki [1979] for epidemiological studies, Kuczera [1982] for hydrological processes, and Suggs and Curran [1983] for compliance with air quality standards), but rarely in biology. This paper explores the use of EB estimates for animal populations, shows how EB estimators are derived, and illustrates how they can offer improved precision of estimators of population size. It is exemplified with survey data on 10 species of waterfowl. Waterfowl surveys are illustrative of animal surveys conducted at regular intervals and therefore are useful to illustrate EB estimators that employ information from previous counts. A simplified version of Efron and Morris' (1972) limited-translation estimator (LTE) also is proffered. This modification

' Manuscript received 15 February 1988; revised 7 July 1988; accepted 29 July 1988. precludes the EB estimate from being markedly different from the estimator customarily calculated.

\section{Survey Methods}

Each May the United States Fish and Wildlife Service (FWS) conducts a survey of the populations throughout the major breeding regions of North America, in order to establish hunting regulations for the following fall and winter hunting season. This sample survey is done in cooperation with the Canadian Wildlife Service and various states and provinces. The area is divided into 49 strata according to similarities in habitats and duck densities (Fig. 1). The sample unit is a transect, a linear route along which an aircraft is flown. Waterfowl are counted, by species, within 200 $\mathrm{m}(1 / 8 \mathrm{mile})$ on either side of the aircraft. To estimate the density of each species along each transect, these counts are divided by the area covered, and then adjusted by independently derived visibility rates that account for ducks that may have been missed by aerial observers (Martin et al. 1979). Information from transects is projected to each stratum, and totals across strata provide the breeding population estimates.

Like many surveys of animal populations, sample counts of waterfowl are subject to large variances, as well as possible biases (Martin et al. 1979). Accordingly, many population estimates derived from May waterfowl surveys are not accurate enough, but improved accuracy through increased sample sizes is not feasible, due to logistic difficulties and the high cost of surveys.

This report proposes and evaluates EB estimators of May populations of 10 duck species: Mallard (Anas platyrhynchos), Gadwall (A. strepera), American Wigeon (A. americana), Green-winged Teal (A. crecca), Blue-winged Teal $(A$. discors), Northern Shoveler $(A$. clypeata), Northern Pintail (A. acuta), Canvasback ( $A y$ thya valisineria), Redhead (A. americana), and Lesser Scaup (A. affinis). 


\section{THE ESTIMATORS}

\section{Derivation of an empirical Bayes estimator}

The EB estimators considered in this report combine information from a sample and from previous surveys. I mostly followed Morris (1983), a recent and comprehensive synthesis that includes suggested variance estimators. The EB procedure may appear convoluted, but is has a simple rationale. Consider a survey that is conducted regularly and provides results that are needed for decision-making. Suppose that for some reason a survey cannot be made, but the decision cannot be put off until the next survey is done. In many situations the missing number would be replaced by the average value during the recent past. That long-term average is thus considered an estimator of the current population, as would the sample estimate, had it been available. Suppose now that the sample estimate is available. Either that value or the long-term average could be used for making the decision. The concept behind the empirical Bayes approach is that a weighted average of the two estimators may be better than either of them alone. The weights used depend on the relative values of the variances of the two estimators.

Although the assumption of normality is made in the following analyses, Efron and Morris (1975) showed that EB estimators are robust to deviations from normality. Moreover, interest lies in the average density of a species over many strata, rather than in a single value, so we can anticipate the central limit effect operating on that average. Further, Johnson (1986) examined some normalizing transformations and found that they offered no general improvement over untransformed variates.

Consider a particular species in some given stratum. We have data for years $i=1, \ldots, I+1$. During each year a count is made on $k$ transects. Let $Y_{i}$ be the average density for the $k$ sample transects in year $i$. Let $\theta_{i}$ be the true density in that stratum for year $i$. Assume that

$$
\begin{gathered}
Y_{i} \mid \theta_{i} \sim N\left(\theta_{i}, V\right) i=1, \ldots, I+1 \\
\text { independently. }
\end{gathered}
$$

That is, given the true density, the sample average density is distributed normally about that true value, with variance $V$. Values from different years are independent. (The veracity of this and other assumptions is of less consequence than the performance of the resulting estimators, which is evaluated later.) Interest lies in estimation of $\theta_{i}$, where $i$ is usually $I+1$, the most recent year.

Assume further that the $\theta_{i}$ are themselves distributed normally, conditional upon parameters $\beta$ and $A$ :

$$
\theta_{i} \mid \beta, A \sim N\left(z_{i}^{\prime} \beta, A\right),
$$

where $z_{i}$ is an $r$-dimensional vector of regression variables, $\beta$ is an $r$-dimensional vector of regression coefficients, and $A$ is the variance.
The parameter of interest can be estimated by either $Y_{i}$ or $z_{i}^{\prime} \hat{\beta}$, where $\hat{\beta}$ is an estimator of $\beta$. A compromise estimator that incorporates both of these estimators and is better than either one alone is

$$
\hat{\theta}_{i}=(1-B) Y_{i}+B\left(z_{i}^{\prime} \hat{\beta}\right),
$$

where $0 \leq B \leq 1$. The optimal value of $B$ depends on the relative values of the variances $V$ and $A$. If $A$ is small compared with $V$, then $z_{i}^{\prime} \hat{\beta}$ estimates $\theta_{i}$ well and $B$ should be near 1 . If $A$ is large relative to $V$, then $Y_{i}$ is the better estimator, and $B$ should be near 0 . That is, greater weight is given the estimator with smaller variance.

If the parameters $V, A$, and $\beta$ were known, then the optimal estimator would be obtained from Eq. 3 with $\beta$ replacing $\hat{\beta}$ and

$$
B=V /(V+A) .
$$

These parameters are usually unknown and must be estimated from the data. Because $Y_{i}$ is a sample mean, its variance $V$ can be estimated from the variance of the constituent observations $\left(s_{i}{ }^{2}\right)$. Since $V$ is assumed constant year-to-year, the average within-year variance can be used. Also, the parameters $A$ and $\beta$ may be estimated from the marginal distribution of $Y_{i}$, which is

$$
Y_{i} \mid \beta, A \sim N\left(z_{i}^{\prime} \beta, V+A\right), \text { independently. }
$$

The estimators

$$
\hat{\beta}=\left(Z^{\prime} Z\right)^{-1} Z^{\prime} Y
$$

and

$$
S=\sum\left(Y_{i}-Z_{i}^{\prime} \hat{\beta}\right)^{2}
$$

are independent and estimate $\beta$ and $A+V$, respectively. Here $Z$ is the $I \times r$ matrix with rows $z_{i}^{\prime}$ and $Y$ is the $I \times 1$ vector of observations $\left\{Y_{i}\right\}$, both based on data from previous years.

Morris (1983) suggested estimating $B$ from

$$
\hat{B}=[(I-r-2) /(I-r)] \hat{V} /\left(\hat{V}+\hat{A}^{+}\right),
$$

where

$$
\hat{A}^{+}=\max [S /(I-r)-\hat{V}, 0] \text {. }
$$

\section{Limited-translation estimators}

Efron and Morris (1972) recognized that, while EB estimators guarantee an overall improvement in estimation of the $\theta_{i}^{\prime}$ s, certain individual $\theta_{i}$ may be poorly estimated, particularly those with unusually large or small values. They developed an estimator that is a compromise between the customary estimator and the EB estimator, called the limited-translation estimator (LTE). Essentially it is the EB estimator, provided that it does not differ from the customary estimator by more than a specified amount. Efron and Morris (1972) developed complicated rules for LTEs, but a simplified LTE was applied in the present study. The limitation was that the EB estimator $\left(\hat{\theta}_{i}^{L}\right)$ could not differ from 
the sample mean $\left(Y_{i}\right)$ by more than, say, one sample standard error $\left(s_{i} / \sqrt{k}\right)$. (Note that this represents a relaxation of the assumption of constant variances in Eq. 1.) Thus

$$
\hat{\theta}_{i}^{L}=(1-\hat{B}) Y_{i}+\hat{B}\left(z_{i}^{\prime} \hat{\beta}\right)
$$

subject to

$$
\left|\hat{\theta}_{i}^{L}-Y_{i}\right| \leq s_{i} / \sqrt{k}
$$

This implies

$$
\begin{aligned}
& s_{i} / \sqrt{k} \geq\left|(1-\hat{B}) Y_{i}+\hat{B}\left(z_{i}^{\prime} \hat{\beta}\right)-Y_{i}\right| \\
& s_{i} / \sqrt{k} \geq \hat{B}\left|z_{i}^{\prime} \hat{\beta}-Y_{i}\right|,
\end{aligned}
$$

which constrains $\hat{B}$ to

$$
\hat{B} \leq\left(s_{i} / \sqrt{k}\right) /\left|z_{i}^{\prime} \hat{\beta}-Y_{i}\right| \text {. }
$$

So for an LT version of an EB estimator, we take

$$
\hat{B}_{L}=\min \left[\hat{B},\left(s_{i} / \sqrt{k}\right) /\left|z_{i}^{\prime} \hat{\beta}-Y_{i}\right|\right] .
$$

\section{Standard errors of EB and LT estimators}

Morris (1983) noted that, as $I$ approaches $\infty$, the variance of $\hat{\theta}_{i}$ approaches

$$
V(1-B),
$$

but questioned the validity of this approximation for small to moderate values of $I$. For this situation he proposed the following variance estimator:

$$
S_{i}^{2}=V[1-(I-r) \hat{B} / I]+\tau\left(Y_{i}-z_{i}^{\prime} \hat{\beta}\right)^{2},
$$

where $\tau$ is the estimated variance of $\hat{B}$ defined by

$$
\tau=[2 /(I-r-2)] \hat{B}^{2} .
$$

For moderately large samples, $\approx 95 \%$ confidence limits are given by

$$
\hat{\theta}_{i} \pm 2 S_{i}
$$

An analogous formula, with $\hat{B}_{L}$ replacing $\hat{B}$ and $\hat{\theta}_{i}{ }^{L}$ replacing $\hat{\theta}_{i}$, was used to calculate standard errors of limited-translations estimators.

\section{Auxiliary information}

Although there are often choices of auxiliary information to use (e.g., Johnson 1986), the present report considers only the average density of a species for a stratum during several (in this case, 10) years previous to the one of concern. The mean of previous years is the usual prior involved in EB estimation. That is, for Eq. 3,

\begin{tabular}{|c|c|c|c|c|c|c|c|}
\hline \multirow[b]{2}{*}{ Year } & \multirow[b]{2}{*}{$i$} & \multirow{2}{*}{$\begin{array}{l}\text { True } \\
\text { value }\end{array}$} & \multicolumn{3}{|c|}{ Transect } & \multirow[b]{2}{*}{$Y_{i}$} & \multirow[b]{2}{*}{$s_{i}^{2} / 3$} \\
\hline & & & 1 & 2 & 3 & & \\
\hline \multicolumn{8}{|c|}{ Density (birds $\left./ 2.59 \mathrm{~km}^{2}\right)^{*}$} \\
\hline 1974 & 1 & 7.91 & 4.68 & 14.23 & 3.69 & 7.53 & 11.29 \\
\hline 1975 & 2 & 7.98 & 10.99 & 12.31 & 5.57 & 9.62 & 4.25 \\
\hline 1976 & 3 & 7.62 & 9.35 & 9.84 & 11.43 & 10.21 & 0.39 \\
\hline 1977 & 4 & 4.08 & 3.65 & 6.25 & 2.19 & 4.03 & 1.41 \\
\hline 1978 & 5 & 9.06 & 7.71 & 5.06 & 6.96 & 6.58 & 0.62 \\
\hline \multicolumn{6}{|c|}{ Average (1974-1977) } & 7.85 & 4.34 \\
\hline
\end{tabular}

$$
z^{\prime} \hat{\beta}=\sum_{j=i-10}^{i-1} Y_{i-j} / 10 .
$$

\section{Example: calculating an EB estimator}

To illustrate the procedure described above, I use 5 yr of mallard densities in a North Dakota area along three transects during 1974-1978 (Table 1). The intention is to use the average density during 1974-1977
TABLE 1. Mallard (Anas platyrhynchos) densities along three transects in North Dakota, 1974-1978 (adapted from Johnson 1981).

* $2.59 \mathrm{~km}^{2}=1 \mathrm{mi}^{2}$.

plus the samples in 1978 to predict the true 1978 value. (True values are ordinarily unknown, but are known in this subsampling illustration.)

In this example, the variable of interest, $Y_{i}$, is the average density along three transects; there are $I=4$ past years; and the regression vector is of dimension $r$ $=1$, containing the mean of previous years. The compromise estimator (Eq. 3) is given by

$$
\hat{\theta_{5}}=(1-\hat{B}) Y_{5}+\hat{B}(\bar{Y}) \text {, }
$$

where $Y_{5}=6.58$ and

$$
\bar{Y}=\left(Y_{1}+Y_{2}+Y_{3}+Y_{4}\right) / 4=7.85 \text {. }
$$

The coefficient $\hat{B}$ is obtained as follows:

$$
S=\sum_{i=1}^{4}\left(Y_{i}-\bar{Y}\right)^{2}=23.40
$$

and $\hat{V}=$ average $\operatorname{var}\left(Y_{i}\right)=4.34$; the finite population correction (e.g., Cochran 1977) is ignored in this example but not elsewhere. Hence

$$
\begin{aligned}
\hat{A}^{+} & =\max [S /(I-r)-\hat{V}, 0] \\
& =\max [23.40 / 3-4.34,0] \\
& =\max [3.46,0] \\
& =3.46 .
\end{aligned}
$$

Then

$$
\begin{aligned}
\hat{B} & =[(I-r-2) /(I-r)] \hat{V} /\left(\hat{V}+\hat{A}^{+}\right) \\
& =[(4-1-2) /(4-1)](4.34) /(4.34+3.46) \\
& =0.185 .
\end{aligned}
$$

Hence

$$
\begin{aligned}
\hat{\theta}_{5} & =(0.815)(6.58)+(0.185)(7.85) \\
& =6.81
\end{aligned}
$$

An approximate standard error can be calculated from Eq. 5 as

$$
S_{5}{ }^{2}=\hat{V}[1-(I-r) \hat{B} / I]+\tau\left(Y_{5}-\bar{Y}\right)^{2} .
$$

Here

$$
\begin{aligned}
\tau & =[2 /(I-r-2)] \hat{B}^{2} \\
& =[2 /(4-1-2)](0.185)^{2} \\
& =0.06845,
\end{aligned}
$$


TABLE 2. Average population size for 1977-1981, root sum of squared errors of customary estimator (RSSE), and percent change in RSSE obtained by empirical Bayes (EB) estimator and limited-translation EB, for all strata.

\begin{tabular}{lcrrr}
\hline & \multirow{2}{*}{$\begin{array}{c}\text { Average } \\
\text { Species }\end{array}$} & & \multicolumn{2}{c}{ Percent change } \\
\cline { 4 - 5 } \multicolumn{1}{c}{ population } & RSSE & EB & LTEB \\
\hline Mallard & 7244 & 856 & -14 & -16 \\
Gadwall & 1583 & 227 & +7 & -13 \\
Am. Wigeon & 3581 & 622 & -2 & -3 \\
G-w. Teal & 2674 & 628 & -12 & -20 \\
B-w. Teal & 4783 & 634 & +12 & -6 \\
N. Shoveler & 2218 & 412 & -2 & -13 \\
N. Pintail & 5447 & 1220 & -33 & -38 \\
Redhead & 872 & 231 & -7 & -14 \\
Canvasback & 641 & 248 & -30 & -29 \\
Scaup & 8354 & 2626 & -53 & -45 \\
Average & 3740 & 770 & -13 & -20 \\
\hline
\end{tabular}

and

$$
\begin{aligned}
\left(Y_{5}-\bar{Y}\right)^{2} & =(6.58-7.85)^{2} \\
& =1.6129
\end{aligned}
$$

so

$$
\begin{aligned}
S_{5}{ }^{2} & =4.34[1-(4-1)(0.185) / 4]+(0.06845)(1.6129) \\
& =3.85 \\
& =(1.96)^{2} .
\end{aligned}
$$

An $\approx 95 \%$ confidence interval for $\theta_{5}$ is given by $\hat{\theta}_{5} \pm$ $2 S_{5}$, or $(2.89,10.73)$.

\section{EVALUATION OF THE EsTimators}

Unlike many investigations of EB estimators, which rely either on theoretical considerations or on limited and possibly artificial data sets, I evaluated the proposed estimators by their performance on substantial sets of actual ecological data. The data consist of densities of 10 species of ducks, adjusted for visibility, for 1967-1981. In several strata too few transects were surveyed to permit a subsampling experiment, so these strata were combined with similar ones nearby, reducing the number of strata to 40 .

\section{The subsampling scheme}

A subsampling approach similar to Johnson (1981) was employed. Data from 1967-1976 were used to calculate the previous mean density for use with the EB estimators; the customary and EB estimators were evaluated on 1977-1981 data. The revised strata contained between 4 and 18 transects. A sample of 2-4 transects was drawn without replacement; a new sample was taken for each year and each stratum. Data from these samples were treated as the observations. The average density from all transects in the stratum for year $i$ is considered $\theta_{i}$, the true value to be estimated. The performance of an estimator is determined largely by how closely it approaches $\theta_{l}$.

\section{Criteria for evaluation}

The primary criterion by which estimators are compared is the root sum of squared errors (RSSE). This quantity is a weighted combination of squared errors, which measure the accuracy of an estimator. The squared error conveniently incorporates both concepts of bias and variance. It is calculated by taking the squared difference between an estimator and the true value, weighting it by the squared area of the stratum $\left(W_{i}^{2}\right)$, and summing over strata; the square root is then taken:

$$
\operatorname{RSSE}=\left[\Sigma_{j} W_{j}^{2}\left(\hat{\theta}_{i j}-\theta_{i j}\right)^{2}\right]^{1 / 2} .
$$

These are then averaged over the years $(i)$ in the evaluation.

A second criterion, involving both an estimator and its estimated standard error, is the average coverage: the fraction of estimates for which the true value is within two standard errors of the estimated value. Nominal coverage for the EB estimators, from Eq. 7, is $\approx 95 \%$. Because the degrees of freedom for the within-years variance estimator are small, however, the Student's $t$ distribution is more appropriate than the normal distribution for determining confidence intervals about the customary estimator. Such intervals would be expected to have lower coverage. Most estimates involve $k=3$ observations, and Student's $t$ statistic with $2 \mathrm{df}$ equals 2.0 for $\alpha=0.18$, so confidence intervals for customary estimators would have nominal coverage of $82 \%$.

I also calculated the average standard error (ASE), the square root of the weighted (by area ${ }^{2}$ ) sum of estimated variances of estimators for each stratum, averaged over the $5 \mathrm{yr}$ in the evaluation period. Small standard errors indicate short confidence intervals, which are desirable if they provide proper coverage.

\section{RESUlts}

\section{Customary estimator}

"True" population numbers of each species, averaged over 1977-1981, are shown in the second column of Table 2. These values are based on all transects in each stratum, weighted by the area of each stratum. Customary estimates, based on average densities during a specific year, were calculated from subsamples of transects. For the 10 species, RSSE averaged $\approx 22 \%$ of the total population, ranging from $12 \%$ for Mallards to $39 \%$ for Canvasbacks.

\section{Ordinary EB estimators}

The EB estimator yielded smaller RSSE than the customary estimator for 8 of the 10 species (Table 2). Improvements were greatest for the scaup, with a 53\% reduction, pintail (33\%), and Canvasback (30\%); it performed the worst for Blue-winged Teal (12\% increase) and Gadwall (7\% increase). For all 10 species, RSSE averaged $13 \%$ smaller for the EB estimator than for 


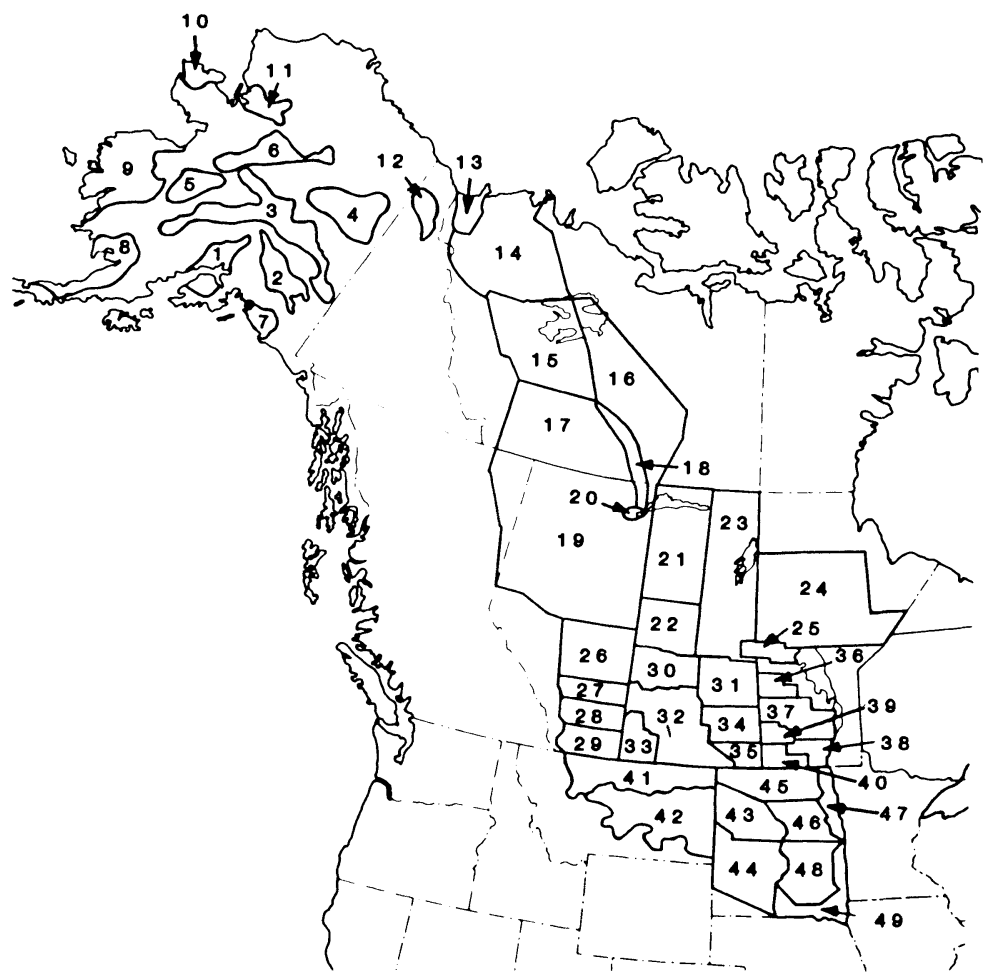

FIG. 1. Strata used in annual surveys of waterfowl in North America.

the customary estimator. The EB estimator had better coverage than the customary estimator (Table 3 ). Only $77 \%$ of the confidence intervals based on the customary estimator included the true value, compared with $90 \%$ for the EB estimator with a standard error calculated from Eq. 5. Not only did EB estimators generally provide greater coverages, but their average standard errors tended to be smaller, by an average of $17 \%$ for the 10 species than for the customary estimator (Table 3).

\section{Limited-translation EB estimators}

Restricting the EB estimate to be within one standard error of the customary estimate, i.e., using a limitedtranslation estimator, generally produced better results than the ordinary EB estimator (Table 2). Average reduction in RSSE for the 10 species, as a percentage of that of the customary estimator, increased from 13 to $20 \%$. More significantly, improvements were noted in the two species for which the ordinary EB estimators performed poorly. The percent change in RSSE improved from +7 to $-13 \%$ for the Gadwall, and from +12 to $-6 \%$ for the Blue-winged Teal; however, the reduction for scaup was not as great ( 45 vs. $53 \%$ ). Coverage of confidence intervals was similar for LT and ordinary EB estimators (Table 3). Again, all were better than those of the customary estimator. Average standard errors were, overall, about the same for LTEB estimators as for ordinary EB estimators (Table 3). All averaged $\approx 15 \%$ smaller than the customary estimator.

\section{Reconstructed population estimates}

The EB estimators developed above can be used to reconstruct population estimates for each species during 1967-1981. I used the limited-translation EB estimator, with the average for the entire 1967-1981 period in place of the average of the previous $10 \mathrm{yr}$. Also, all transects were included, not only those selected in the subsampling experiment.

One major difference between the customary estimates and the LTEB estimates is apparent (Fig. 2). The curves based on EB values are considerably smoother; they display far less year-to-year variation than the customary estimates. Much of the variation in customary estimates is not biologically feasible. For example, the $59 \%$ increase in Green-winged Teal from 1977 to 1978, and the 35\% decrease in Northern Shovelers from 1972 to 1973 are unlikely fluctuations. The LTEB estimates presented in Fig. 2 are probably more representative of population changes that actually occurred during 1967-1981.

\section{Discussion}

Overall, the non-limited-translation EB estimators were superior in RSSE to the customary estimator. The EB estimator gave an average reduction of RSSE of $\approx 13 \%$, even though it was slightly poorer than the customary estimator for Gadwall and Blue-winged Teal. It was anticipated that the EB estimators would fare 

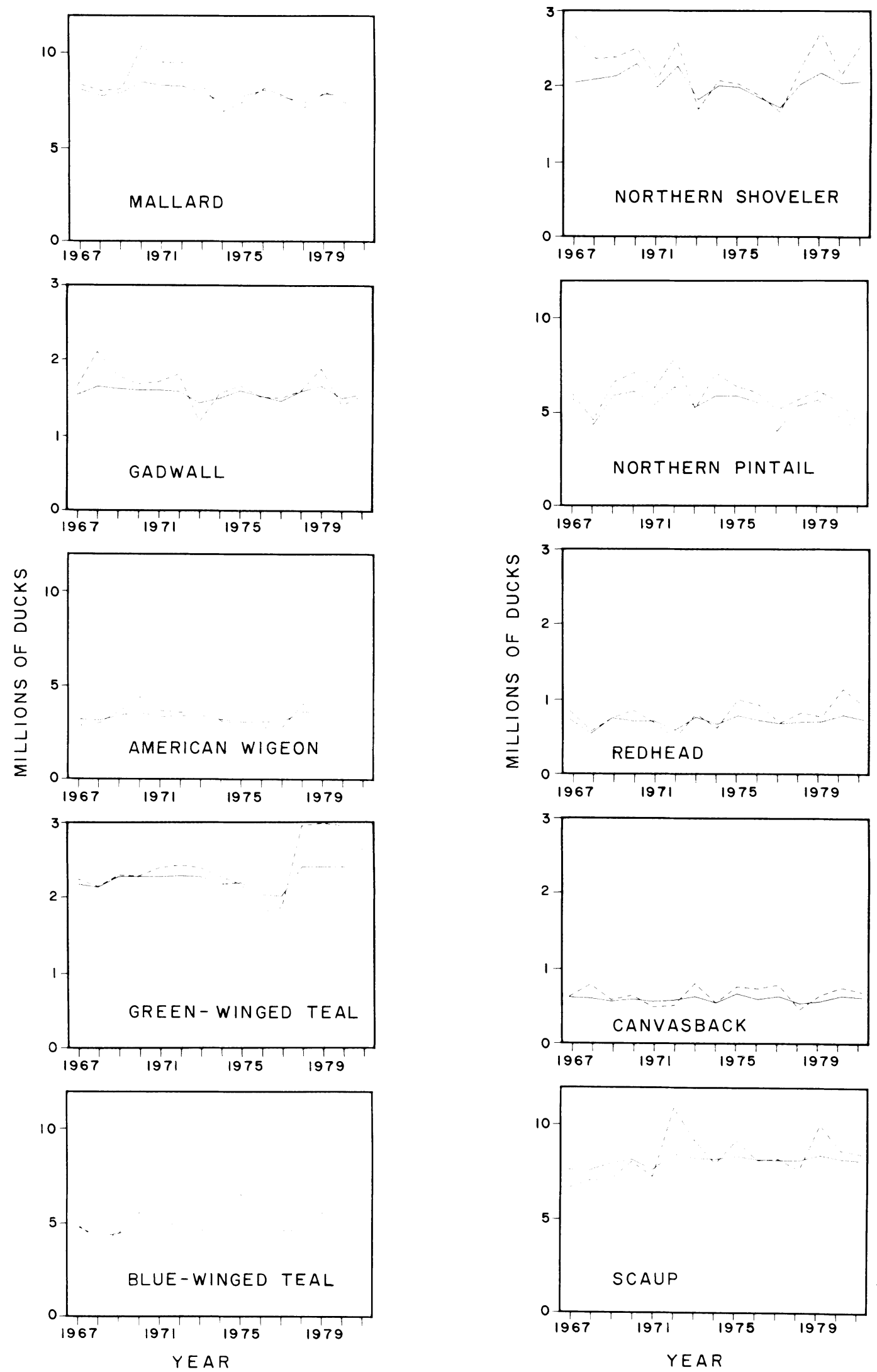

FIG. 2. Customary estimates (----) and limited-translation empirical Bayes estimates ( - ) of population sizes of 10 species of ducks during 1967-1981. 
TABLE 3. Average standard error (ASE) of customary estimator and percent change in value obtained by empirical Bayes (EB) and limited-translation EB (LTEB) estimators, and coverage of confidence intervals, for all strata.

\begin{tabular}{|c|c|c|c|c|c|c|}
\hline \multirow[b]{3}{*}{ Species } & \multirow[b]{3}{*}{ ASE } & \multirow{2}{*}{\multicolumn{2}{|c|}{ Percent change ASE }} & \multicolumn{3}{|c|}{ Coverage } \\
\hline & & & & \multirow{2}{*}{$\begin{array}{l}\text { Customary } \\
\text { estimator }\end{array}$} & \multirow[b]{2}{*}{ EB } & \multirow[b]{2}{*}{ LTEB } \\
\hline & & EB & LTEB & & & \\
\hline Mallard & 902 & 108 & 107 & 0.795 & 0.935 & 0.930 \\
\hline Gadwall & 324 & 80 & 84 & 0.830 & 0.925 & 0.935 \\
\hline Am. Wigeon & 703 & 72 & 73 & 0.740 & 0.860 & 0.855 \\
\hline G-w. Teal & 562 & 84 & 85 & 0.770 & 0.925 & 0.920 \\
\hline B-w. Teal & 850 & 105 & 104 & 0.830 & 0.920 & 0.920 \\
\hline N. Shoveler & 400 & 102 & 112 & 0.755 & 0.870 & 0.870 \\
\hline N. Pintail & 1295 & 76 & 77 & 0.740 & 0.930 & 0.935 \\
\hline Redhead & 274 & 73 & 71 & 0.775 & 0.860 & 0.860 \\
\hline Canvasback & 261 & 68 & 73 & 0.760 & 0.895 & 0.905 \\
\hline Scaup & 2929 & 68 & 71 & 0.715 & 0.905 & 0.900 \\
\hline Average & 850 & 83 & 85 & 0.771 & 0.902 & 0.903 \\
\hline
\end{tabular}

worse than the customary ones for a few combinations of species, strata, and years, but the consistently poorer performance across all strata for $5 \mathrm{yr}$ for these two species was unexpected. For several other species in some strata, improvements were smaller than anticipated.

These results prompted a closer inspection of the data. For example, EB estimates of Northern Pintail in stratum 32 were far from the actual counts. A detailed examination showed that pintail densities (birds per square kilometre) during the 1967-1976 period averaged 9.1 birds $/ \mathrm{km}^{2}$ and ranged from 4.5 to 14.4 birds $/ \mathrm{km}^{2}$ for all 14 transects. Densities during the 1977-1981 evaluation period averaged only 3.8 pintails $/ \mathrm{km}^{2}$ and ranged from 1.8 to 6.3 pintails $/ \mathrm{km}^{2}$. The reason for this $60 \%$ average decline is unknown, but it is clear that using data from the 1967-1976 period to predict densities during 1977-1981 is inopportune. Other combinations of species and strata in which EB estimators performed poorly also provided indications that the assumptions of this EB procedure, notably the constancy of variance in Eq. 1, may have been violated. This suggestion gave support for the limited-translation version of $\mathrm{EB}$ estimation, which essentially does not give full weight to the EB estimator.

Limited-translation EB estimators performed well for estimating duck populations from recurring surveys, in contrast to Carter and Rolph (1974), who found LT versions not worth the additional computations they require. Limited-translation EB estimators yielded RSSE reductions of $\approx 20 \%$ and, more importantly, improvements were consistent among species. The disadvantage of the limited-translation procedure is that it does not take full advantage of the EB assumptions when they are true, so improvements may not be as great as they could be (e.g., scaup).

Average standard errors for both ordinary and limited-translation EB estimators were smaller than those for the customary estimator. Nonetheless, the EB estimators provided much greater coverage than did customary ones. The greater coverage of EB estimators was partly due to the greater accuracy of those estimators and partly due to the pooling involved in estimates of standard errors, which essentially increased the degrees of freedom from the nominal two to a greater value.

The adoption of empirical Bayes estimators should result in more accurate estimates of waterfowl population size for most species in most years. Moreover, confidence intervals would have better coverage. It is anticipated that EB methods would yield similar improvements for surveys of other animal populations. The methodology is likely to provide greatest gains when standard errors of the estimates are large relative to actual changes in the population; in those situations year-to-year changes are difficult to detect and annual estimates are imprecise.

The EB approach presented here is most directly applicable to recurring surveys that are statistically independent and provide an estimate of standard error. The waterfowl survey used as an example is one such application. The method could be applied as well to the Breeding Bird Survey, which is conducted annually in the United States and Canada (Robbins et al. 1986). Routes (samples) within a stratum could be used to provide an estimated standard error $(\hat{V})$, and year-toyear variability could be used for the estimate $A$.

Many recurring surveys do not furnish estimates of standard errors, usually because replications are lacking. For these, EB estimators could be developed by performing some replications in $1 \mathrm{yr}$, just to estimate $V$, and assuming the same value held in other years. Even a subjective estimate of $V$ could yield improved estimators. Returning to the data (Table 1) used in the example, suppose the estimate of the within-year variation $(\hat{V}=4.34)$ was unavailable, and we used instead an arbitrary value $V=1.00$. Then the ensuing estimate of $\theta_{5}$ would be 6.63 , which is closer to the true value than the customary estimate is, but not as close as the EB estimate that uses the appropriate value of $\hat{V}$.

Other extensions are feasible. For example, many surveys are designed to compare indices of animal 
abundance from occasion to occasion. This is perhaps the most common application of the Breeding Bird Survey, as well as the Common Bird Census in Great Britain (Mountford 1982). Methods for statistically estimating trends in such indices are complicated (Geissler and Noon 1981, Mountford 1982), in part because of the imbalance in the design caused by not having all samples included in all years. An EB approach to this problem would likely develop a model, not for the indices themselves, but for the ratio of change from one occasion to the next. Another extension of EB involves population estimates obtained from markrecapture techniques (Otis et al. 1978). These do not immediately fit the EB framework described here, because estimates from different occasions are not independent. An EB formulation might involve a distribution for capture probabilities and use EB estimates for those.

Other methods have been used to smooth data from recurring animal surveys. Most prominent is the taking of moving averages, a technique rooted in time series analysis but often employed to smooth suspiciously erratic estimates. More rigorous are methods such as the smoothing of scatterplots (Cleveland 1979), which offers a robust estimator of a line through a scatter of points, such as population estimates for each occasion. EB methodology has a firmer theoretical basis than most of these procedures, because of its realistic biological underpinnings. As Krebs (1978: 289) noted, "Population densities are continually changing, but their values tend to vary about a characteristic density." This is a key statement, which provides the rationale for empirical Bayes procedures in biology, and suggests why EB estimators offer improvements over customary estimators.

\section{ACKNOWLEDGMENTS}

The author is grateful to R. S. Pospahala for providing the data and for support, and to J. Sedransk, T. L. Shaffer, and the referees for comments on earlier drafts of the report.

\section{LITERATURE Cited}

Carter, G. M., and J. F. Rolph. 1974. Empirical Bayes methods applied to estimating fire alarm probabilities. Journal of the American Statistical Association 69:880-885.

Cleveland, W. S. 1979. Robust locally weighted regression and smoothing scatterplots. Journal of the American Statistical Association 74:829-836.

Cochran, W. G. 1977. Sampling techniques. Third edition. John Wiley \& Sons, New York, New York, USA.

Cooperrider, A. Y., R. J. Boyd, and H. R. Stuart, editors. 1986. Inventory and monitoring of wildlife habitat. United States Department of the Interior, Bureau of Land Management, Denver, Colorado, USA.
Eberhardt, L. L. 1978. Transect methods for population studies. Journal of Wildlife Management 42:1-31.

Efron, B., and C. Morris. 1972. Limiting the risk of Bayes and empirical Bayes estimators-Part II: the empirical Bayes case. Journal of the American Statistical Association 67: 130-139.

Efron, B., and C. Morris. 1975. Data analysis using Stein's estimator and its generalizations. Journal of the American Statistical Association 70:311-319.

Geissler, P. H., and B. R. Noon. 1981. Estimates of avian population trends from the North American breeding bird survey. Pages 42-51 in C. J. Ralph and J. M. Scott, editors. Estimating numbers of terrestrial birds. Studies in Avian Biology 6.

Harris, E. K., and G. Shakarki. 1979. Use of the population distribution to improve estimation of individual means in epidemiological studies. Journal of Chronic Diseases 32: 233-243.

Johnson, D. H. 1981. Improved population estimates through the use of auxiliary information. Pages 436-440 in C. J. Ralph and J. M. Scott, editors. Estimating numbers of terrestrial birds. Studies in Avian Biology 6.

- 1986. Empirical Bayes estimates of breeding populations of North American ducks. Dissertation. North Dakota State University, Fargo, North Dakota, USA.

Krebs, C. J. 1978. Ecology. Second edition. Harper \& Row, New York, New York, USA.

Kuczera, G. 1982. Combining site-specific and regional information: an empirical Bayes approach. Water Resources Research 18:306-314.

Martin, F. W., R. S. Pospahala, and J. D. Nichols. 1979 Assessment and population management of North American migratory birds. Pages 187-239 in J. Cairns, G. P. Patil, and W. E. Waters, editors. Environmental biomonitoring, assessment, prediction, and management-certain case studies and related quantitative issues. International Co-operative Publishing House, Fairland, Maryland, USA.

Morris, C. N. 1983. Parametric empirical Bayes inference: theory and applications (with discussion). Journal of the American Statistical Association 78:47-65.

Mountford, M. D. 1982. Estimation of population fluctuations with application to the common bird census. Applied Statistics 31:135-143.

Otis, D. L., K. P. Burnham, G. C. White, and D. R. Anderson. 1978. Statistical inference from capture data on closed animal populations. Wildlife Monographs 62.

Ralph, C. J., and J. M. Scott, editors. 1981. Estimating numbers of terrestrial birds. Studies in Avian Biology 6.

Robbins, C. S., D. Bystrak, and P. H. Geissler. 1986. The breeding bird survey: its first fifteen years, 1965-1979. United States Fish and Wildlife Service Resource Publication 157.

Seber, G. A. F. 1982. The estimation of animal abundance. Second edition. Macmillan, New York, New York, USA.

Suggs, J. C., and T. C. Curran. 1983. An empirical Bayes method for comparing air pollution data to air quality standards. Atmospheric Environment 17:837-841.

Wilcox, R. R. 1977. Estimating the likelihood of false-positive and false-negative decisions in mastery testing: an empirical Bayes approach. Journal of Educational Statistics 2: 289-307. 